\title{
Effets Antihypertensifs Des Extraits Aqueux Et Éthanolique Des Graines Fermentées De Parkia Biglobosa (Mimosaceae) Chez Les Rats
}

\section{Coulibaly Seydou Ouolouho}

Etudiant-Doctorant, Laboratoire de Pharmacodynamie-Biochimique Ufr Biosciences, Université Félix Houphouët-Boigny, Abidjan, Côte d'Ivoire

\section{Ouattara Abou}

Enseignant-Chercheur, Département de Biochimie-Microbiologie Université Jean Lorougnon Guédé, Daloa, Côte d'Ivoire

\section{Ouattara Karamoko \\ Coulibaly Adama}

Enseignant-Chercheur, Laboratoire de Pharmacodynamie-Biochimique Ufr Biosciences, Université Félix Houphouët-Boigny, Abidjan, Côte d'Ivoire

Doi: 10.19044/esj.2017.v13n36p162 URL:http://dx.doi.org/10.19044/esj.2017.v13n36p162

\section{Abstract}

High blood pressure is a major cardiovascular risk factor in the occurrence of stroke, heart failure, kidney failure, and coronary heart disease. They are regarded as the leading causes of death worldwide. The aim of this study was to provide a scientific and pharmacological basis for the traditional use of Parkia biglobosa in the traditional treatment of arterial hypertension. Here, we first looked for certain mineral elements in the fermented seeds of P. biglobosa "soumara". Subsequently, we evaluated the hypotensive and antihypertensive effects of the aqueous and ethanolic extracts from these fermented seeds of P. biglobosa in normotensive and hypertensive rats. The cardiovascular parameters studied were systolic blood pressure (SBP), diastolic blood pressure (DBP), and heart rate (HR). The results showed that "soumara" is rich in calcium, potassium and magnesium, but low in sodium. Also, plant extracts at doses between 1000 and $2000 \mathrm{mg} /$ $\mathrm{kg}$ bw induced a dose-dependent decrease in cardiovascular parameters in healthy rats. The treatment of rats made hypertensive with adrenaline by the ethanolic extract at a dose of $1500 \mathrm{mg} / \mathrm{kg}$ bw induced a dose-dependent decrease in cardiovascular parameters until normalization. The aqueous and ethanolic extracts of "soumara" acted in the same way as Nifédipine ${ }^{\circledR}$, which is a calcium channel blocker. 
Keywords: Antihypertensive, hypotensor, seeds fermented of néré, $P$. biglobosa

\section{Résumé}

L'hypertension artérielle est un facteur de risque cardio-vasculaire majeur dans la survenue d'accidents vasculaires cérébraux, d'insuffisance cardiaque, d'insuffisance rénale et de maladies coronaires qui représentent les principales causes de décès dans le monde. L'objectif de ce travail était d'apporter une base scientifique et pharmacologique à l'usage traditionnelle de Parkia biglobosa dans le traitement traditionnel de l'hypertension artérielle. Nous avons d'abord recherché certains éléments minéraux dans les graines fermentées de P. biglobosa «soumara ». Par la suite, nous avons évalué les effets hypotenseurs et antihyeprtensifs des extraits aqueux et éthanolique issus de ces graines fermentées de $P$. biglobosa sur des rats normotendus et hypertendus. Les paramètres cardiovasculaires étudiés ont été la pression artérielle systolique (PAS), la pression artérielle diastolique (PAD) et la fréquence cardiaque (FC). Les résultats ont montré que le «soumara» est riche en calcium, potassium et magnésium mais pauvre en sodium. Aussi, les extraits végétaux aux doses comprises entre 1000 et $2000 \mathrm{mg} / \mathrm{kg}$ pc ont induit une diminution dose-dépendante des paramètres cardiovasculaires chez les rats sains. Le traitement des rats rendus hypertendus avec l'adrénaline par l'extrait éthanolique à la dose $1500 \mathrm{mg} / \mathrm{kg}$ pc a induit une diminution dose-dépendante des paramètres cardiovasculaires jusqu'à leur normalisation. Les extraits aqueux et éthanolique du soumara ont agi de la même façon que la Nifédipine ${ }^{\circledR}$ qui est un inhibiteur calcique.

Mots-clés: antihypertensive, hypotenseur, graines fermentées de néré, $P$. biglobosa

\section{Introduction}

L'hypertension artérielle est un facteur de risque cardio-vasculaire majeur dans la survenue d'accidents vasculaires cérébraux, d'insuffisance cardiaque, d'insuffisance rénale et de maladies coronaires qui représentent les principales causes de décès dans le monde (OMS, 2009). Selon l'OMS (2013), plus d'un adulte sur trois est hypertendu à partir de l'âge de 25 ans touchant ainsi environ un milliard de personnes. Les projections sur la prévalence de l'HTA sont inquiétantes, car pour 2025, une augmentation de $60 \%$ de la prévalence chez les adultes au niveau mondiale est attendue, soit 1,56 milliard de nouvelles personnes hypertendues dont près de $80 \%$ dans les pays en développement (Kearney et al., 2005). En Afrique subsaharienne, c'est 80 millions d'hypertendus qui sont répertoriés et les projections reposant sur des données épidémiologiques suggèrent que ce chiffre passera à 150 millions en 2025 (Fourcade et al., 2007). Cependant, le coût des 
traitements efficaces contre 1'hypertension artérielle reste assez élevé et l'accès aux soins est souvent difficile pour les pays en développement créant ainsi un véritable problème de santé publique.

Face à une telle situation, les populations des pays défavorisés se sont orientées vers l'utilisation des plantes médicinales qui occupent une place prépondérante pour se soigner. Afin d'aider ces populations à tirer un réel profit de l'usage des plantes médicinales, l'OMS encourage la mise au point de batterie de protocoles standards pour la réalisation des enquêtes ethnobotaniques et les travaux expérimentaux permettant de valider les utilisations traditionnelles (Kim, 2005). A cet effet, une enquête ethnobotanique menée par notre laboratoire a permis la sélection de nombreuses plantes à vertus thérapeutiques intéressantes contre l'hypertension. Parmi ces plantes, figure Parkia Biglobosa qui est une espèce de la famille des Mimosaceae. Les études antérieures ont révélé que les extraits aqueux de l'écorce de Parkia Biglobosa ont des propriétés antihypertensives (Yomalan et al., 2008). En Côte d'Ivoire, les graines de cette plante «néré» ayant subi une fermentation sont appelées communément «soumara » en langue «malinké» et constituent une source de protéines (Odunfa, 1983). Ce condiment « soumara » est réputé avoir des propriétés antihypertensives selon ses consommateurs. Le présent travail se propose donc d'étudier les effets des extraits aqueux et éthanolique des graines fermentées du néré « soumara » sur les paramètres cardiovasculaires chez les rats rendus hypertendus.

\section{Matériels et Méthode \\ Matériel Végétal}

Il est constitué des graines fermentées de Parkia Biglobosa «soumara». Ce produit est obtenu par un ensemble d'opérations unitaires technologiques (Figure 1) qui présente trois étapes essentielles dont la première cuisson, la deuxième cuisson et la fermentation (N'Dir et al., 1994).

La première cuisson des graines crues (Figure 2) est longue et dure 15 à 24 heures. Elle a pour but de ramollir le tégument séminal des graines. Après cette première étape essentielle, les graines sont décortiquées et lavées à grande eau puis subissent une seconde cuisson moins longue que la première (1 à 2 heures). Les amandes obtenues sont par la suite essorées, triées et mises à fermenter entre 28 à $40^{\circ} \mathrm{C}$ pendant trois jours. Les graines fermentées sont enfin récupérées et séchées au soleil pour obtenir le soumara (Figure 3). 


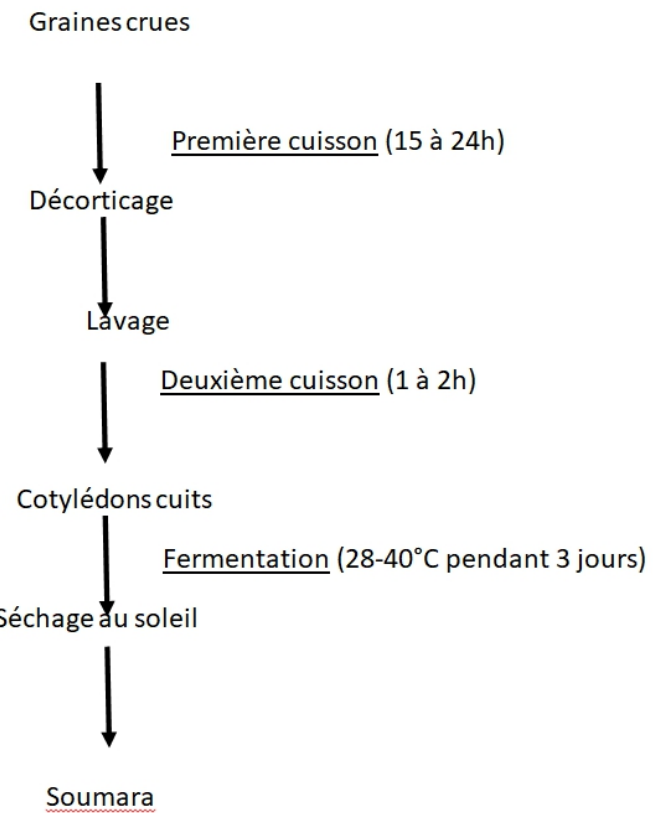

Figure 1. Diagramme de fabrication du soumara (N'Dir et al., 1994).

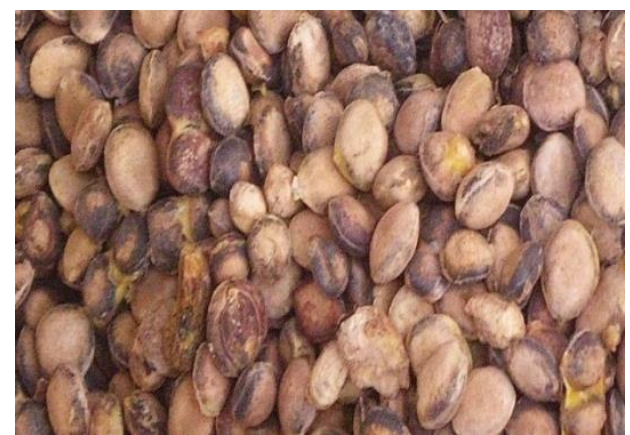

Figure 2. Graines crues de P.biglobosa «néré » (source: Coulibaly.S.O)

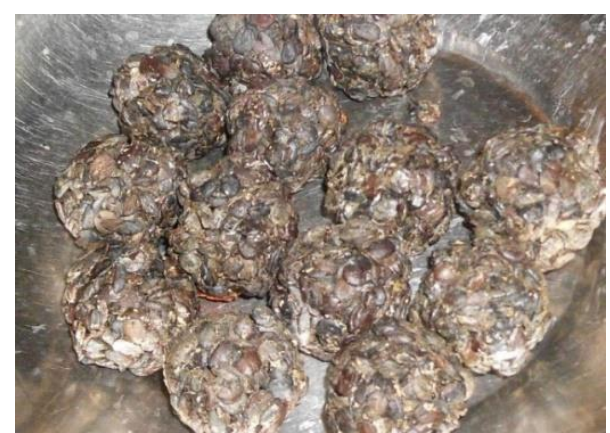

Figure 3. Graines fermentées de P.biglobosa (source: Coulibaly.S.O)

\section{Matériel Animal}

Des rats blancs albinos, mâles et femelles, de souches Wistar pesant entre $130 \mathrm{~g}$ et $180 \mathrm{~g}$ ont été utilisés dans cette étude.

\section{Méthode}

\section{Préparation des Différents Extraits Végétaux}

Deux (02) échantillons de $500 \mathrm{~g}$ de soumara issus du mode de production de la Figure 1 ont été préalablement séchés à la température ambiante du laboratoire puis broyés et réduits en poudre dans un mortier. Par 
la suite, $250 \mathrm{~g}$ de poudre de soumara ont été dissouts dans $500 \mathrm{~mL}$ d'eau distillée puis homogénéisés sous agitation magnétique pendant 24 heures à $25^{\circ} \mathrm{C}$ à l'aide d'un agitateur magnétique. L'homogénat obtenu a été filtré successivement deux fois sur du coton hydrophile puis une fois sur du papier Whatman $\mathrm{n}^{\circ} 2$. Le filtrât obtenu est évaporé à l'aide d'une étuve de type Med Center Venticell à $50^{\circ} \mathrm{C}$ pour donner une poudre qui constitue l'extrait aqueux.

La même opération a été réalisée en utilisant en lieu et place de l'eau distillée de l'éthanol $70 \%$. Cependant à la différence, le volume du filtrat hydro-alcoolique obtenu est d'abord réduit à l'aide d'un évaporateur rotatif de type Büchi à la température de $60^{\circ} \mathrm{C}$. Ensuite, le reste du filtrât est évaporé à l'aide d'une étuve de type Med Center Venticell à $50^{\circ} \mathrm{C}$ pour donner une poudre qui représente l'extrait éthanolique 70\%.Tous les extraits bruts végétaux ainsi constitués sont conservés au réfrigérateur $\left(4^{\circ} \mathrm{C}-6^{\circ} \mathrm{C}\right)$ jusqu'à leur utilisation pour les différents essais.

\section{Analyse Quantitative de Quelques Éléments Minéraux des Graines Fermentées}

$10 \mathrm{~g}$ d'échantillon de graines fermentées sont placés dans une capsule d'incinération initialement tarée. La capsule contenant l'échantillon est mise dans un four à moufle et l'échantillon est incinéré à $550 \pm 15^{\circ} \mathrm{C}$ pendant 12 heures. La capsule contenant la cendre est retirée du four à moufle, refroidi dans un dessiccateur et pesé (AOAC, 1990).

La microanalyse des cendres obtenues a été faite selon la méthode de la Spectrométrie à Diffusion d'Energies (EDS). Elle a été réalisée par l'appareil MEB-EDS à pression variable de la D.C.A.R (MEB FEG Supra 40 VP Zeiss). Ce MEB est équipé d'un détecteur de rayons-X (OXFORD Instruments) X-MAX SDD relié à une plateforme de microanalyseur EDS (Inca Dry Cool, sans azote liquide). Les rayons-X émises dépendent de la nature de l'échantillon. Pour identifier la composition chimique des éléments l'appareil effectue une mesure de l'énergie de transition des électrons au niveau des nuages électroniques des séries $\mathrm{K}$; $\mathrm{L}$ et $\mathrm{M}$ des atomes de l'échantillon.

\section{Criblage Phytochimique des Différents Extraits}

Dans cette étude, plusieurs tests ont été effectués afin de mettre en évidence la présence ou non de différentes familles de métabolites secondaires. Ce sont, entre autres, les alcaloïdes (réactions de Dragendorff Valser-Mayer), les polyphénols (Bonga et al., 1995), les tanins (Nemlin \& Brunel, 1995), les flavonoïdes (Bowman \& Rand, 1980), les saponosides (Lebeau \& Janot, 1956), les polyterpènes et les stérols (réaction de Liebermann) puis les glycosides cardiotoniques (Shivakumar et al., 2011). 


\section{Mesure de L'effet Hypotenseur et Antihypertenseur des Différents Extraits}

La pression artérielle (PA) des rats a été enregistrée par la méthode indirecte à l'aide du dispositif d'enregistrement Visitech BP 2000 (France). Ce système permet de mesurer simultanément la $\mathrm{PA}$ de quatre rats. Le système d'enregistrement Visitech BP 2000 est constitué par une plate-forme chauffante avec deux boutons, un verre à fond carré, un thermomètre de contrôle de la température et un ordinateur portable avec le logiciel BP 2000 Blood Pressure analysis version 2004 permettant de visualiser les variations de la pression artérielle et la fréquence.

Ainsi, avant chaque enregistrement, la veine caudale est dilatée grâce au passage des rats dans une potence de chauffage pendant cinq minutes. Les rats sont ensuite placés dans les cellules de contention et le capteur de pression inséré sur la queue. Le signal de pression artérielle détecté émet des variations de pressions qui sont visualisées sur l'écran de l'ordinateur de même que les valeurs de pressions et de fréquence cardiaque correspondantes (Boga, 2015).

\section{Evaluation de L'effet Hypotenseur des Différents Extraits Sur les Rats Normotendus}

Vingt-huit (28) rats de souche Wistar âgés de 2 à 3 mois et pesant entre 130 à $180 \mathrm{~g}$ ont été utilisés pour cette expérimentation. Les rats sont répartis en 7 lots de 4 rats chacun. Le lot témoin (lot 1) a reçu par gavage 1 $\mathrm{mL}$ d'eau distillée en une seule dose. Les lots 2, 3 et 4 ont reçu par gavage et en une seule prise $1 \mathrm{~mL}$ de l'extrait aqueux de P.Biglobosa respectivement aux doses de 1000, 1500 et $2000 \mathrm{mg} / \mathrm{kg}$ pc. Une heure après l'administration de l'extrait, la pression artérielle est mesurée à l'aide du dispositif Visitech BP 2000 dont le mode de fonctionnement est décrit plus haut. Les rats des lots 5,6 et 7 quant à eux ont reçu l'extrait éthanolique dans les conditions similaires.

Evaluation de L'effet Antihypertenseur des Différents Extraits et de la Nifédipine ${ }^{\circledR}$ Sur les Rats Rendus Hypertendus

Trente-deux (32) rats âgés de 2 à 3 mois dont les poids varient entre 130 à $180 \mathrm{~g}$ ont été utilisés. Ces rats ont été répartis en deux groupes. Un groupe constitué du lot témoin (4 rats) et un autre groupe test de 28 rats.

Les rats du lot témoin ont reçu par voie orale de l'eau distillée en fonction de leur poids corporel pendant 14 jours (temps d'expérimentation). Ceux du groupe essai, ont reçu par injection intrapéritonéale de l'adrénaline (ADR) à la dose de $3,6.10^{-1} \mathrm{mg} / \mathrm{kg}$ pc (Omale \& Ebimala, 2011; Umang et al., 2012). 
Après l'installation de 1'hypertension artérielle (HTA), le groupe test de 28 rats hypertendus a été ensuite reparti en 7 lots de 4 rats chacun. Le traitement a été fait comme suit:

- $\quad$ Le lot $_{1}$ HypNT (lot constitué de rats hypertendus non traités ou lot contrôle positif): après l'induction de l'hypertension artérielle, les rats de ce lot n'ont subi aucun traitement pendant les 6 jours qu'a duré le traitement des autres lots essais. Ces rats ont reçu de l'eau distillée pendant la durée de l'expérience.

Le $\operatorname{lot}_{2}$ Nif 10: après l'induction de HTA, les rats de ce lot ont reçu par gavage $(1 \mathrm{~mL})$ de la Nifédipine $\AA$ à la dose de $10 \mathrm{mg} / \mathrm{kg}$ pc pendant 6 jours (durée du traitement).

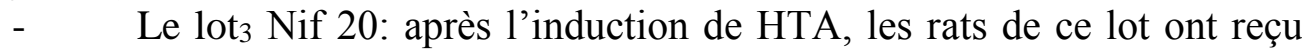
par gavage $(1 \mathrm{~mL})$ de la Nifédipine ${ }^{\circledR}$ à la dose de $20 \mathrm{mg} / \mathrm{kg}$ pc pendant 6 jours.

- $\quad$ Le lot 4 Eth 1000: après l'induction de HTA, les rats de ce lot ont reçu par gavage $(1 \mathrm{~mL})$ de l'extrait éthanolique à la dose de $1000 \mathrm{mg} / \mathrm{kg} \mathrm{pc}$ pendant 6 jours.

- $\quad$ Le lot ${ }_{5}$ Eth 1500: après l'induction de HTA, les rats de ce lot ont reçu par gavage $(1 \mathrm{~mL})$ l'extrait éthanolique à la dose de $1500 \mathrm{mg} / \mathrm{kg}$ pc pendant 6 jours.

- Le lot ${ }_{6} \mathrm{Aq}$ 1500: après l'induction de HTA, les rats de ce lot ont reçu par gavage $(1 \mathrm{~mL})$ de l'extrait aqueux à la dose de $1500 \mathrm{mg} / \mathrm{kg}$ pc pendant 6 jours.

- $\quad$ Le $\operatorname{lot}_{7} \mathrm{Aq}$ 2000: après l'induction de HTA, les rats de ce lot ont reçu par gavage $(1 \mathrm{~mL})$ de l'extrait aqueux à la dose de $2000 \mathrm{mg} / \mathrm{kg}$ pc pendant 6 jours.

La pression artérielle (PA) a été mesurée quotidiennement jusqu’à la normalisation des paramètres cardiovasculaires.

\section{Analyse Statistique des Résultats}

L'analyse statistique de l'ensemble des résultats exprimés en moyennes accompagnées des écarts type (Moyenne \pm ET), a été réalisée suivant l'analyse des variances (ANOVA ONE WAY). Lorsqu'il y a plus de deux échantillons à analyser, il convient d'utiliser une analyse de variance (ANOVA) adapté. Une ANOVA à un facteur (univarié) suivie du test de Tukey a été choisie. Les différences entre les moyennes ont été déterminées selon le test de comparaison multiple de Tukey grâce au logiciel Graph Pad Prism 5.0 (Microsoft U.S.A). Ces différences sont considérées significatives lorsque $\mathrm{p}<0,01$. 


\section{Résultats et Discussion}

\section{Analyse Quantitative de Quelques Éléments Minéraux des Graines Fermentées}

Dans la présente étude, les graines fermentées de $P$. biglobosa (soumara) ont présenté des teneurs variables en minéraux. En effet, l'incinération de ces graines fermentées a donné des teneurs de 13,82 et 8,64 $\mathrm{g} / 100 \mathrm{~g}$ de matière sèche respectivement pour le calcium et le potassium. Ces graines fermentées ont enregistré également des teneurs en magnésium de $6,52 \mathrm{~g} / 100 \mathrm{~g}$ de matière sèche contre $0,61 \mathrm{~g} / 100 \mathrm{~g}$ de matière sèche pour le sodium (Tableau 1). Ainsi, les résultats de cette étude montrent que le soumara est riche en calcium, potassium et magnésium mais pauvre en sodium. Déjà des études effectuées par Lamboni et al. (1999) démontraient que des échantillons de soumara avaient des teneurs élevées en magnésium. Ce condiment pourrait être donc recommandé dans un régime DASH (Dietary Approches to Stop Hypertension) qui est une approche diététique pour arrêter l'hypertension. En effet, un régime DASH est faible en matières grasses totales, en graisses saturées et en cholestérol, et riche en calcium, en potassium, en magnésium et en fibres.

Tableau 1. Teneurs en minéraux des graines fermentées de P.Biglobosa

\begin{tabular}{cc} 
Minéraux Recherchés & Teneurs $(\mathrm{g} / 100 \mathrm{~g}$ de matière sèche $)$ \\
Calcium & 13.82 \\
Potassium & 8.64 \\
Sodium & 0.61 \\
Magnésium & 6.52 \\
\hline
\end{tabular}

\section{Criblage Phytochimique}

Le criblage phytochimique des différents extraits issus des graines fermentées de P.Biglobosaa révélé la présence de plusieurs grands groupes chimiques tels que les flavonoïdes, les polyphénols, les saponosides, les tanins, les glycosides cardiotoniques et les sucres réducteurs. Ce screening basé en général sur les tests de précipitation et de coloration en tube montre une diversité de groupes chimiques au sein d'un même extrait. D'une façon générale, les deux extraits étudiés renferment plus de composés phénoliques. Aussi des faibles teneurs en saponoside, sucres réducteurs et glycoside cardiotonique ont été détectées dans ces deux extraits préparés. Toutefois, comme le montre le Tableau 2, ces extraits ne contiendraient pas d'alcaloïdes, de stérols et les polyterpènes. L'activité antihypertensive de la plupart de ces composés notamment les flavonoïdes, a été déjà démontrée par plusieurs chercheurs (N'guessan et al., 2011; Mamyrbekova-Bekro et al., 2012). 
Tableau 2. Composition chimique des différents extraits des graines fermentées de P.Biglobosa

\begin{tabular}{|c|c|c|c|}
\hline $\begin{array}{l}\text { Groupes Chimiques } \\
\text { Recherchés }\end{array}$ & Tests & Extrait Aqueux & Extrait Éthanolique \\
\hline Flavonoïdes & Test à la cyanidine & ++ & ++ \\
\hline Polyphénols & Test de chlorure ferrique & ++ & ++ \\
\hline Saponosides & Test de mousse $(>1 \mathrm{~cm})$ & + & + \\
\hline \multirow{2}{*}{ Tanins } & Test de Stiasny & + & + \\
\hline & Test de chlorure de fer & + & + \\
\hline \multirow{2}{*}{ Alcaloïdes } & Test de Dragendorff & - & - \\
\hline & Test de Mayer & - & - \\
\hline $\begin{array}{c}\text { Glycosides } \\
\text { cardiotoniques }\end{array}$ & Test de Keller Killiani & + & + \\
\hline Stérols et terpènes & Test de Liebermann & - & - \\
\hline Sucres réducteurs & Test de Fehling & + & + \\
\hline
\end{tabular}

$-:$ absence, $+:$ présence, $++:$ abondance

\section{Effet Hypotenseur des Différents Extraits Sur les Rats Normotendus}

La Figure 4 montre l'effet des extraits aqueux et éthanolique des graines fermentées de P.biglobosa sur la pression artérielle systolique (PAS) chez les rats normotendus. L'extrait aqueux a provoqué une diminution significative de la PAS de $119,5 \pm 3,78 \mathrm{mmHg}$ chez les rats normotendus à respectivement $112,9 \pm 4,40 ; 100,5 \pm 0,19$ et $97,70 \pm 0,97 \mathrm{mmHg}$. Ce qui correspond à des pourcentages de diminution respectifs de 5,52\%;15,90\% et $18,24 \%$. Cependant, l'extrait éthanolique a entrainé une diminution plus importante de la PAS que l'extrait aqueux. Avec cet extrait, la PAS est passée de $119,5 \pm 3,78 \mathrm{mmHg}$ chez les rats normotendus à respectivement $106,3 \pm 1,96 ; 97,82 \pm 0,35$ et $86,12 \pm 1,90 \mathrm{mmHg}$. Ce qui correspond également à des pourcentages de diminution respectifs de $11,05 \% ; 18,14 \%$ et $27,93 \%$.

Aussi, la Figure 5 présente les effets des extraits aqueux et éthanolique des graines fermentées de P.biglobosa sur la pression artérielle diastolique (PAD) chez les rats normotendus. Les extraits aqueux ont entrainé une réduction significative de la PAD de $104,5 \pm 2,17 \mathrm{mmHg}$ à respectivement $100,6 \pm 1,01,97,93 \pm 0,36,77,31 \pm 0,67 \mathrm{mmHg}$. Des pourcentages de réduction respectifs de $11,05 \%, 18,14 \%$ et $27,93 \%$ ont été enregistrés pour cet extrait avec des concentrations variables. Aussi, les extraits éthanoliques ont provoqué des réductions plus importantes de la $\mathrm{PAD}$ que les extraits aqueux de 104,5 $\pm 2,17 \mathrm{mmHg}$ à respectivement 88,12 $\pm 0,39 ; 77,36 \pm 0,65$ et $67,59 \pm 0,71 \mathrm{mmHg}$. En pourcentage, cela équivaut respectivement à $15,67 \%, 25,97 \%$ et $35,32 \%$. 
La Figure 6 présente les effets des extraits aqueux et éthanolique des graines fermentées de $P$. biglobosa sur la fréquence cardiaque (FC) chez les rats normotendus. L'extrait aqueux a entrainé une baisse significative de la $\mathrm{FC}$ de $250,3 \pm 3,83 \mathrm{mmHg}$ à respectivement 235,7 $\pm 1,67,224 \pm 2,65$ et $218,3 \pm 1,45 \mathrm{mmHg}$. Cela correspond aux pourcentages de réduction respective de $5,83 \%, 10,50 \%$ et $12,78 \%$. La réduction de la FC obtenue par l'extrait hydroalcoolique est plus importante passant de 250,3 $\pm 3,83 \mathrm{mmHgà}$ $189 \pm 3,51 \mathrm{mmHg}$.

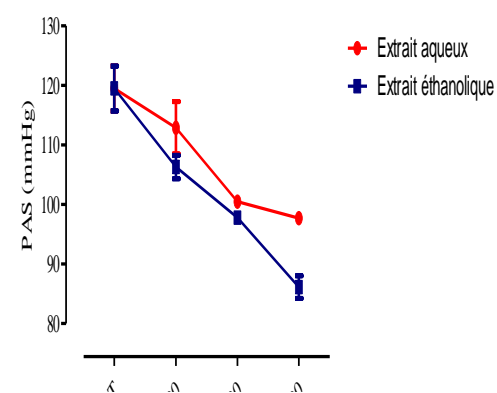

Figure 4. Effet hypotenseur des extraits aqueux et éthanolique des graines fermentées de P.biglobosa sur la pression artérielle systolique des rats normotendus

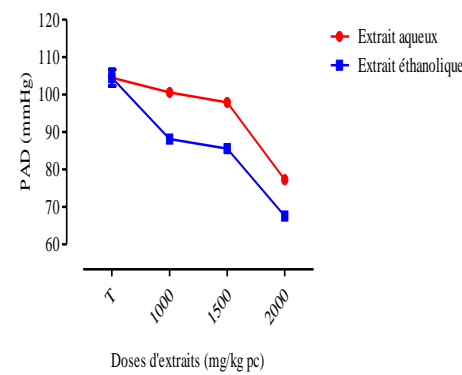

Figure 5. Effet hypotenseur des extraits aqueux et éthanolique des graines fermentées de P.biglobosa sur la pression artérielle diastolique des rats normotendus

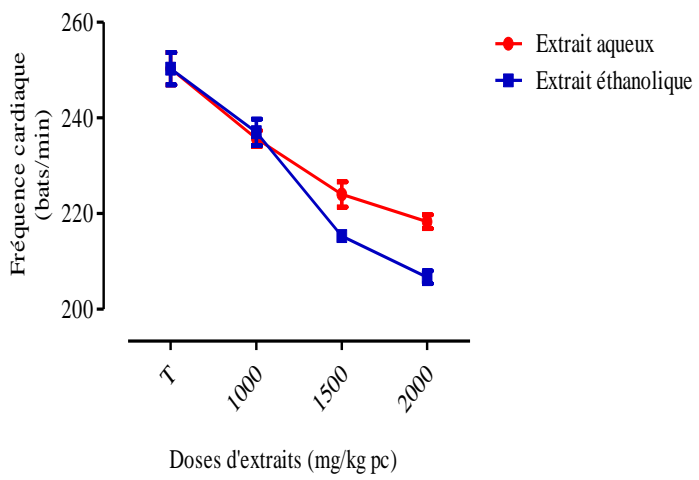

Figure 6. Effet hypotenseur des extraits aqueux et éthanolique des graines fermentées de P.biglobosa sur la fréquence cardiaque des rats normotendus

\section{Effet Antihypertenseur des Différents Extraits et de La Nifédipine ${ }^{\circledR}$ Sur les Rats Rendus Hypertendus}

Les résultats des effets antihypertenseurs des extraits aqueux et éthanolique des graines fermentées du P.Biglobosa et de la Nifédipine ${ }^{\circledR}$ sur les paramètres cardiovasculaires chez les rats rendus hypertendus sont représentés par les figures 7 ( $7 \mathrm{a}, 7 \mathrm{~b}$ et $7 \mathrm{c})$. 
L'injection intrapéritonéale de $1 \mathrm{~mL}$ de l'ADR à la dose de 3,6.10${ }^{1} \mathrm{mg} / \mathrm{kg}$ pc aux rats normotendus, a entrainé une élévation significative de la pression artérielle. La pression artérielle systolique (PAS) est passée de $119,5 \pm 3,78 \mathrm{mmHg}$ chez les rats témoins normotendus à 181,2 $\pm 2,21$ $\mathrm{mmHg}$, soit une augmentation de 51,63\%. La pression artérielle diastolique (PAD) est passée de $104,5 \pm 2,17 \mathrm{mmHg}$ à $144,7 \pm 2,03 \mathrm{mmHg}$, ce qui représente également une augmentation de $38,47 \%$.

L'adrénaline (ADR) a également provoqué une augmentation significative de la fréquence cardiaque (FC) et de la pression artérielle moyenne (PAM) qui sont passées respectivement à 250,3 $\pm 3,39$ battements par minutes (bpm) et 109,5 $\pm 2,7 \mathrm{mmHg}$ à $156,87 \pm 2,09 \mathrm{mmHg}$, soit des pourcentages d'augmentation respectifs de $21,85 \%$ et $43,26 \%$. Cependant l'administration des extraits aqueux, éthanolique et de la Nifédipine ${ }^{\circledR}$ aux rats rendus hypertendus a réduit significativement $(\mathrm{p}<0,01)$ les valeurs des paramètres cardiovasculaires.

En effet l'administration de l'extrait éthanolique à la dose de 1000 $\mathrm{mg} / \mathrm{kg}$ pc a entrainé une baisse significative de la PAS, de la PAD et de la FC chez les rats traités. Ces paramètres sont passés respectivement à 144,8 \pm $1,86 \mathrm{mmHg}$, à $112,4 \pm 0,09 \mathrm{mmHg}$ et à $249,7 \pm 0,67 \mathrm{bpm}$, donnant ainsi des pourcentages de réduction respectifsde $20,09 \%, 22,25 \%$ et $18,13 \%$. A la dose de $1500 \mathrm{mg} / \mathrm{kg} \mathrm{pc}$, cet extrait éthanolique a induit une réduction plus importante de la PAS, la PAD et la FC qui furent respectivement de 32,45 $\%, 27,02 \%$ et $18,59 \%$.

Cependant, le traitement des rats rendus hypertendus par l'extrait aqueux a montré une diminution moins prononcée que l'extrait éthanolique. Ainsi l'extrait aqueux à la dose de $1500 \mathrm{mg} / \mathrm{kg}$ pc a diminué la PAS, la PAD et la FC respectivement de $15,29 \%, 21,49 \%$ et $15,74 \%$. A une dose de $2000 \mathrm{mg} / \mathrm{kg} \mathrm{pc}$, ce même extrait aqueux a induit des inhibitions de $26 \%$ de la PAS, de $22,18 \%$ de la PAD et de $17,15 \%$ de la FC.

La Nifédipine ${ }^{\circledR}$ à la dose de $10 \mathrm{mg} / \mathrm{kg}$ pc a baissé la PAS de $26 \%$, la PAD de $22,18 \%$ et la FC de $17,15 \%$. A la dose de $20 \mathrm{mg} / \mathrm{kg}$ pc, la réduction a été de $32,23 \%$ pour la PAS, de $29,23 \%$ pour la PAD et de $19,44 \%$ pour la FC. Par ailleurs, l'extrait éthanolique à la dose de $1500 \mathrm{mg} / \mathrm{kg}$ pc et la Nifédipine ${ }^{\circledR}$ à la dose de $20 \mathrm{mg} / \mathrm{kg}$ pc ont ramené la PAS, la PAD et la FC à leurs valeurs normales.

Des résultats similaires ont été déjà obtenus par d'autres chercheurs (Assane et al., 1993; Bonnah et al., 1998). En effet, ces auteurs ont démontré un effet d'atténuation de l'hypertension artérielle des graines crues de $P$. biglobosa respectivement chez des lapins et chez des cobayes.

L'effet des extraits de P.Biglobosa sur les paramètres cardiovasculaires étudiés pourrait être expliqué par plusieurs mécanismes. Cependant, la Nifédipine ${ }^{\circledR}$ (antihypertenseur de référence utilisé dans cette 
étude) est un antagoniste calcique qui agit en bloquant les canaux calciques voltage-dépendants (Leonetti et al., 2002). En effet, le calcium intervient dans la contraction des muscles lisses, l'inhibition de son entrée va donc entrainer au niveau des fibres cardiaques une réduction de la contractilité et un ralentissement de la conduction auriculo-ventriculaire, une réduction de l'automaticité sinusale (Varon \& Mark, 2002) et une relaxation des fibres lisses ce qui aura pour conséquence une baisse de la pression artérielle (Hauhouot-Attoungbré et al., 1997).

Les extraits de $P$. Biglobosa auraient agi en utilisant le même mécanisme d'action que la Nifédipine ${ }^{\circledR}$. En effet, l'échantillon de «soumara» utilisé contient du calcium et magnésium qui sont antagonistes. Ainsi, une élévation de la concentration en magnésium dans le milieux extracellulaire provoquerait une diminution de la concentration du calcium dans les cellules musculaires lisses et pourrait entrainer une baisse de certains paramètres cardiovasculaires (Yang et al., 2000). Certains auteurs ayant travaillé sur l'extrait aqueux de P. Biglobosa ont montré que les principes actifs hypotenseurs étaient au moins de deux types dont l'un de type bêta bloquant et l'autre de type anticalcique (Yomalan et al., 2008).

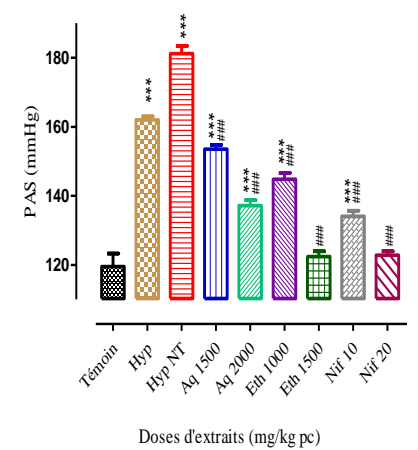

Figure 7a. Effets sur la PAS

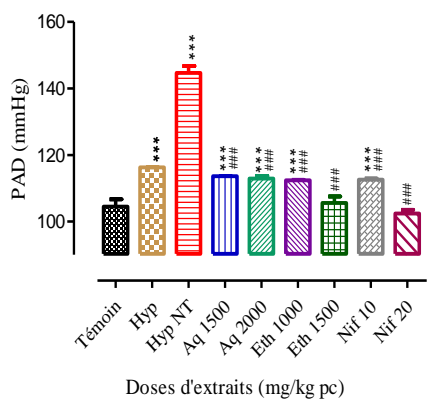

Figure 7b. Effets sur la PAD

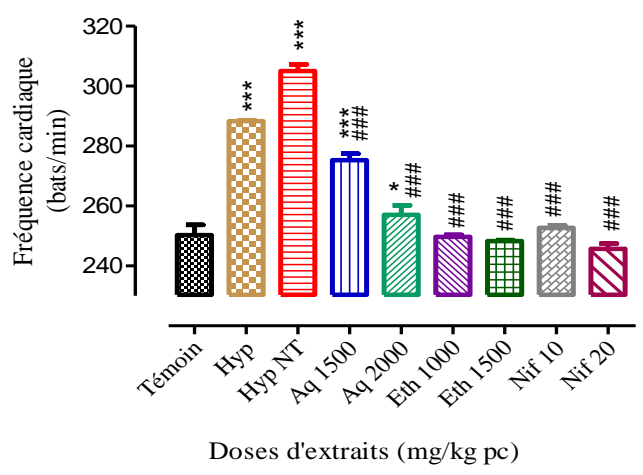

Figure 7c. Effets sur la fréquence cardiaque 
Figure 7. Effets antihypertenseurs des extraits aqueux, éthanolique des graines fermentées de P.Biglobosa et de la Nifédipine ${ }^{\circledR}$ sur les paramètres cardiovasculaires chez les rats rendus hypertendus par l'adrénaline

$P A S:$ pression artérielle systolique, PAD : pression artérielle diastoliqu), FC : fréquence cardiaque

Chaque barre représente la moyenne $\pm E S M, n=4$. ${ }^{*} * *<0,01$; différence significative par rapport au lot témoin. \#\#\# $P<0,01$, différence significative par rapport au lot malade non traité,

Hyp $=$ lot hypertendu, Hyp NT = lot malade non traité (témoin positif); Aq $1500=$ lot hypertendu traité avec l'extrait aqueux à la dose de $1500 \mathrm{mg} / \mathrm{kg} \mathrm{pc}, \mathrm{Aq} 2000=\mathrm{lot}$ hypertendu traité avec l'extrait aqueux à la dose de $2000 \mathrm{mg} / \mathrm{kg} \mathrm{pc}$; Eth $1000=$ lot hypertendu traité avec l'extrait éthanolique à la dose de $1000 \mathrm{mg} / \mathrm{kg}$ pc, Eth $1500=$ lot hypertendu traité avec l'extrait éthanolique à la dose de $1500 \mathrm{mg} / \mathrm{kg} \mathrm{pc}$; Nif $10=\mathrm{lot}$ hypertendu traité avec la nifédipine à la dose de $10 \mathrm{mg} / \mathrm{kg}$ pc et Nif $20=$ lot hypertendu traité avec la niféfdipine à la dose de $20 \mathrm{mg} / \mathrm{kg} \mathrm{pc}$.

\section{Conclusion}

De nombreux travaux de recherches ont été menés sur la fermentation et la valeur nutritionnelle des graines fermentées de Parkia biglobosa "soumara". Cette étude situe sur les propriétés antihypertensives des graines fermentées de cette plante. L'activité antihypertensive de l'extrait hydro-alcoolique issu de ces graines fermentées est plus accrue que celle de l'extrait aqueux.

Par ailleurs, d'autres études sont actuellement en cours pour évaluer l'effet de ce condiment sur les paramètres biochimiques et hématologiques chez le rat.

\section{References:}

1. AOAC (1990). Official methods of analysis. Association of Official Analytical Chemists Ed., Washington DC, 684 p

2. Assane, M., Baba-Moussa, R., Bassene, E. \& Sere, A. (1993). Etude de l'action antihypertensive des graines de Parkia biglobosa (JACQ). Benth chez le rat. Dakar-Méd 38 : 49-54.

3. Boga, G. L. (2015). Evaluation des effets antihypertenseurs de l'extrait total dichlorométhane-éthanol des feuilles de Morinda morindoides (Baker) Milne-Redh (Rubiaceae) (EDTE) sur l'hypertension artérielle induite chez le rat. Thèse de doctorat (Pharmacologie des substances naturelles), Université Félix Houphouët-Boigny (Côte d'Ivoire), 144p.

4. Bonga, G. M., Vangah-Manda, M., De Souza, C. \& Guédé-Guina, F. (1995). Mise en évidence des phytostéroides antifongiques contre cryptococus neoformans. Revue Médecine Pharmacologique Africaine, 9 : 21-30. 
5. Bonnah, B., Aklikolou, K.A., Akpagana, K. \& Gbeassor, M. (1998). Contribution à l'étude des propriétés pharmacologiques des extraits hydro-alcooliques des graines de Parkia biglobosa. Sciences et Médecine, Revue CAMES 12-15.

6. Bowman, W. C. \& Rand, M. J. (1980). Textbook of pharmacology. Black well Scientific Publication, Second Edition, 5, 1928 p.

7. Fourcade, L., Paule, P. \& Mafort, B. (2007). Hypertension artérielle en Afrique Subsahérienne, actualité et perspectives. Revue générale Med Trop, 67: 559-567.

8. Kearney, D. M., Whelton, M., Reynolds, K., Muntner, P., Whelton, P. K. \& He, J. (2005). Global burden of analysis of worldwide data. Lancet; 365: 217-23.

9. Kim H-S. (2005). Do not put too much value on conventional medicines. J. Ethnopharmacol., 100: 37-39.

10. Leonetti, G., Magnami, B., Pessina, A.C., Rappelli, A., Trimarco, B. \& Zanchetti, A. (2002). Tolerability of long -term treatment with lercanidipine versus amlodine and lacidipine in elderly hyperthensives. Am. J. Hypertens. 15: 932-40.

11. Lebeau, P. \& Janot, M. (1956). Traité de pharmacie chimique. Masson, Paris. France, 5, 838 p. Tome 2, 3, 4 et 5.

12. Lamboni, C., Monkpoh, K., Konlani, S. \& Doh, A. (1999). Caractéristiques alimentaires «Tonou », condiment à base de soja ou de graines de néré. Méd Nut. 2 : 50-65.

13. Mamyrbekova-Bekro, J.A., Boua, B.B., Kouassi, K.C. \& Békro, YA. (2012). Sur l'analyse qualitative et pharmacologique de 2 plantes antihypertensives utilisées à N'gramanssabo en Côte d'Ivoire. Revue « Nature \& technologie » B-Sciences Agronomiques et Biologiques, $\mathrm{n}^{\mathrm{o}} 8,11: 12-2$.

14. Nemlin, J. \& Brunel, J. E. (1995). Fascicule de Travaux Pratiques de Matière Médicale ( $3^{\mathrm{e}}$ année). Université Nationale de Côte-d'Ivoire. Faculté de Pharmacie. Département de Pharmacognosie. Laboratoire de Physiologie, $47 \mathrm{p}$.

15. N'Guessan, H.A., Dago, D.C.C., Namyrbekova-Békro, J.A. \& Békro, Y-A. (2011). CCM d'extraits Sélectifs de 10 Plantes Utilisées Dans le Traitement Traditionnel de l'hypertension artérielle en Côte d'Ivoire. European Journal of Scientific Research, 580:585-576.

16. Odunfa, S. A. (1983). Carbohydrate changes in fermenting locust bean (Parkia biglobosa). Chem Microbiol Technol Lebensm; 10: 1257.

17. Ognatan, K., Adi, K., Lamboni, C., Damorou, J.M., Aklikokou, K.A., Gbeassor, M. \& Guillard, J.C. (2011). Effect of Diatery Intake of Fermented Seeds of Parkia biglobosa (Jacq) Benth (African Locust 
Bean) on Hypertension in Bogou and Goumou-Kope Aereas of Togo. Trop J Pharm Res, $10: 603$.

18. Omale, J., Etubi, A.F. \& Ebiloma, G.U. (2011). Antihypertensive effect of methanol extract of Napoleona imperialis (P. beauv) in adrenalin induced hypertensive albino rats. Int. J. Biochem. Res. Rev., 1: 47-57.

19. OMS (2009). Global health risk: mortality and burden of disease attributable to selected risk (http://www.who.in/healthinfo/global_burdendisease/GlobalhealthRis k_report_full.pdf)

20. OMS (2013). Journée mondiale de la santé : mesurez votre tension artérielle pour rester en bonne santé. (http://www.who.int/mediacentre/news/releases/2013/world health day 20130403/fr).

21. Umang, H. J. \& Tejas, H.G., Tusharbindu, R. D. \& Pravin, R. T. (2012). Evaluation of antihypertensive activity of Evolvulus alsinoides in adrenaline induced hypertensive rats. Int $J$ Pharm Pharm Sci, 4: 194-198.

22. Varan, J. \& Mark, P.E. (2002). Clinical review. The management of hyper-tensive crises. Crit care, 7(5): 374-384.

23. Yomalan, K., Kadjo, J.A., Kouakou, J-C.A., Méa, A., Sémi, A., Néné, B. \& Ehouan, E. (2008). Effet antihypertensif d'un extrait aqueux d'écorce de tronc de Parkia Biglobosa (mimosaceae) sur la pression artérielle du lapin. Sciences et Nature, 2: 133-143.

24. Yang, Z.W., Gebrewold, A., Nowakowski, M., Altura, B.T. \& Altura, B.M. (2000). $\mathrm{Mg}^{2+}$-induced endothelium-dependent relaxation of blood vessels and blood pressure lowering: role of NO. American Journal of Physiology-ReguPhisiol, 3: 628-639. 